\title{
TV/Series
}

$10 \mid 2016$

Guerres en séries (II)

\section{War Without End: le devoir de mémoire dans Babylon 5 (PTEN>TNT, 1993-1998)}

\section{Florent Favard}

\section{(2) OpenEdition}

\section{Journals}

Édition électronique

URL : http://journals.openedition.org/tvseries/1855

DOI : 10.4000/tvseries.1855

ISSN : 2266-0909

\section{Éditeur}

GRIC - Groupe de recherche Identités et Cultures

\section{Référence électronique}

Florent Favard, «War Without End : le devoir de mémoire dans Babylon 5 (PTEN>TNT, 1993-1998)», TV/ Series [En ligne], 10 | 2016, mis en ligne le 29 novembre 2016, consulté le 20 avril 2019. URL : http:// journals.openedition.org/tvseries/1855; DOI : 10.4000/tvseries.1855

Ce document a été généré automatiquement le 20 avril 2019

\section{(c) (1) () $\Theta$}

TV/Series est mis à disposition selon les termes de la licence Creative Commons Attribution - Pas d'Utilisation Commerciale - Pas de Modification 4.0 International. 


\title{
War Without End : le devoir de mémoire dans Babylon 5 (PTEN>TNT, 1993-1998)
}

\author{
Florent Favard
}

1 Diffusée en syndication sur le réseau PTEN de 1993 à 1997, puis sur la chaîne du câble TNT pour sa dernière saison en 1998, Babylon 5 a représenté une révolution narrative, esthétique et technologique, rarement égalée dans l'histoire de la télévision américaine, «rompant avec la tradition» des séries de science-fiction épisodiques qui ne se structuraient pas autour d'arcs narratifs à long terme ${ }^{1}$. Si la recherche sur les séries télévisées - notamment française - cite régulièrement Hill Street Blues (NBC, 1981-1987) comme un des creusets de l'hybridation des formes épisodiques et feuilletonnantes qui caractérise la « complexité narrative ${ }^{2}$ » des séries contemporaines, Babylon 5 est une autre œuvre majeure ayant bousculé les conventions esthétiques d'alors ${ }^{3}$. Située « à des annéeslumière de tout ce qui se fai[sai]t à la télévision ", comme le proclamait son matériel promotionnel, elle a marqué le genre de la science-fiction en déployant des conflits d'envergure galactiques entre de multiples civilisations, à travers le prisme de la station spatiale diplomatique éponyme.

Babylon 5 possède la particularité d'avoir articulé sur le long terme une «intrigue continue » qui évoque celle qui lie entre eux les différents volumes d'un cycle littéraire ${ }^{4}$; une intrigue macroscopique qui dépasse l'échelle des épisodes, des saisons, pour structurer un récit sur cinq années. Un récit, de surcroît, que son créateur J. Michael Straczynski, figure auctoriale maîtresse ${ }^{5}$, a toujours affirmé avoir planifié longtemps avant l'entrée en production de la série ${ }^{6}$, dont il a écrit près de 90 de ses 110 épisodes. Un récit avec un début, un milieu et une fin, explorant un réseau de conflits interconnectés dans un monde fictionnel extrêmement dense et complexe, étendu dans l'espace et le temps, menacé par une civilisation millénaire belliqueuse. Les voix-off qui rythment les génériques de chaque saison évoquent un regard en arrière sur des faits passés, et la série se présente comme chronique de ces faits, de leur déclenchement, leur enchaînement, 
leur conclusion. Ainsi que le rappelle Éric Dufour, Babylon 5 opère comme toutes les œuvres de science-fiction qui « thématisent leur monde comme monde »:

[...] le récit de S-F met en évidence la profonde intrication des dimensions technique, scientifique, politique, morale, juridique et religieuse, insistant par-là même sur le fait qu'elles sont entremêlées et donc dépendantes les unes des autres ${ }^{7}$.

3 Tout récit de science-fiction nous renvoie à son contexte socio-culturel de production et de réception, aussi éloigné puisse-t-il paraître des étoiles, civilisations extraterrestres et autres vaisseaux spatiaux. Au-delà d'une intrigue de plus en plus feuilletonnante, la série est ainsi dotée d'une matrice ${ }^{8}$ reformulant sans cesse un questionnement sur le devoir de mémoire, la responsabilité de chacun(e) face à l'Histoire', et notre point de vue mouvant sur le passé.

Mon objectif est ici d'explorer la façon dont Babylon 5 use de guerres futuristes et interstellaires pour mieux nous renvoyer à notre propre rapport à l'Histoire. Après un tour d'horizon de la série qui facilitera les développements ultérieurs, je m'engagerai dans une analyse narratologique principalement thématique et me pencherai en premier lieu sur la façon dont la série construit ses divers conflits en écho avec le public étatsunien auquel elle s'adresse en priorité, tout en arborant des prétentions universalistes ; je m'intéresserai ensuite à la façon dont Babylon 5 sollicite constamment des problématiques liées au devoir de mémoire et à l'écriture de l'Histoire.

\section{Un monde fictionnel complexe}

Babylon 5 se trouve être le nom d'une station diplomatique terrienne en orbite autour de la lointaine planète Epsilon III. Le récit se déroule au XXIII ${ }^{\mathrm{e}}$ siècle, et l'endroit accueille les ambassadeurs et ambassadrices des grandes civilisations de la galaxie, ainsi que les représentant(e)s de peuples encore minoritaires. Si le monde fictionnel s'étend sur de nombreuses planètes, la station va vite devenir le nexus d'une guerre interstellaire contre les Ombres, un ancien ennemi bien décidé à imposer son règne.

Le monde fictionnel de la série est fractionné entre cinq civilisations principales, dont les Terriens qui sont représentés par la hiérarchie militaire de la station. À la tête de Babylon 5 se succèdent en effet le commandant Jeffrey Sinclair (Michael O'Hare, saison 1), le capitaine John Sheridan (Bruce Boxleitner, saisons 2 à 4), et la colonelle Elizabeth Lochley (Tracy Scoggins, saison 5). Sous leurs ordres, on trouve une grande partie du casting, de la commandante en second Susan Ivanova (Claudia Christian) au chef de la sécurité Michael Garibaldi (Jerry Doyle), en passant par le médecin-chef Steven Franklin (Richard Biggs).

7 Cette omniprésence militaire évoque d'autres programmes contemporains de Babylon 5, exploitant une «science-fiction militariste »: Space: Above and Beyond (Fox, 1995-1996) et ses soldats d'élites; seaQuest DSV (NBC, 1993-1996) et ses garde-côtes du futur ; ou encore la franchise Stargate (Showtime>Sci Fi, 1997-2007, et dérivées) - une tendance dont Mehdi Achouche note qu'elle est aussi représentée au cinéma dans les années $1990^{10}$. Le fait que la station diplomatique, bâtie par la Terre, soit sous supervision militaire traduit aussi le malaise qui plane encore entre les Terriens et les Minbaris, avec qui les premiers étaient en guerre dix ans plus tôt : la station diplomatique est perçue comme «le dernier espoir de paix » (ainsi que le rappelle le générique des trois premières saisons), et la paix est sans cesse montrée comme fragile, voire illusoire, au fil de la série. 
8 Les Minbaris sont une civilisation plus évoluée que les Terriens, mais présentent une structure sociétale ternaire qui évoque la division médiévale en trois corps, religieux, guerrier et ouvrier. C'est l'ambassadrice Delenn (Mira Furlan) qui les représente sur Babylon 5. Les Centauris sont quant à eux montrés comme d'égocentriques impérialistes se drapant dans le prestige de leur gloire passée - une puissance économique qu'ils ont longtemps tiré de l'asservissement et de la colonisation d'autres peuples, notamment les Narns. Les Centauris évoquent ainsi tout autant la Rome Antique que l'Empire napoléonien ou la Russie des tsars, caractérisés en cela par leur ambassadeur, Londo Mollari (Peter Jurasik), un opportuniste imbu de lui-même qui passera un pacte faustien avec les Ombres et comprendra trop tard le prix à payer.

9 Les Narns, longtemps réduits en esclavage par les Centauris, affichent quant à eux une apparence reptilienne, et leur ambassadeur G'Kar (Andreas Katsulas) apparaît initialement comme aussi sournois et belliqueux que Londo Mollari. Mais lorsque les masques tombent, et qu'au fil de la série les Centauris lancent une nouvelle guerre contre les Narns, ces derniers révèlent leur vraie nature : celle d'un peuple longtemps asservi par la colonisation, opprimé, et pourtant plein d'espoir. Les Vorlons, enfin, sont une civilisation mystérieuse que même les Minbaris, qui collaborent parfois avec eux, ne connaissent pas vraiment. Dissimulés sous de lourdes combinaisons cachant leur véritable apparence, ils dévoilent bientôt, derrière l'illusion d'un interventionnisme bienveillant, un paternalisme et une rigueur qui n'a d'égal que celle de leurs ennemis millénaires, les Ombres. Là où les Vorlons s'attendent à ce que toutes les civilisations de la galaxie leur obéissent aveuglément en échange de leur protection, les Ombres souhaitent attiser des conflits internes, usant de la loi du plus fort pour faire émerger des civilisations plus puissantes et belliqueuses au milieu du chaos.

Le monde fictionnel de Babylon 5 possède une structure qui, au fil de la série, passe de dyadique, celle du mythe classique, à hybride, celle du monde moderne, si l'on suit la typologie de Lubomír Doležel ${ }^{11}$ : cela signifie que, d'un monde où les domaines " naturel » et « surnaturel » sont clairement séparés, on passe à un autre où les frontières deviennent floues, fondé sur la dialectique entre visible et invisible. Babylon 5 n'est certes pas une série de fantasy, où l'apport du mythe classique serait plus directement observable, mais elle appartient à une veine de la science-fiction nourrie de fantasy, où les anciennes civilisations (les Vorlons, les Ombres) possèdent une technologie si évoluée qu'elle est « indiscernable de la magie", pour reprendre la troisième loi de l'écrivain Arthur C. Clarke $^{12}$. Au fil des saisons, les Terriens et Minbaris notamment vont transgresser les espaces interdits des Vorlons et des Ombres, et les nombreux conflits de la série vont faire éclater cet ordre millénaire. Il devient vite évident, au fil de la série, que la voix-off du générique ne se trompe pas en présentant régulièrement la station comme un morceau d'Histoire, « à l'aube du Troisième Âge de l'humanité » : la dynamique imprimée au monde fictionnel dans son ensemble est celle d'une révolution qui va le marquer à jamais. Une perspective facilitée par les prolepses opérées par quelques épisodes, qui contemplent avec recul - et souvent, de façon partielle et partiale - l'enchevêtrement de conflits narrés par le récit premier ${ }^{13}$.

\section{Des conflits interconnectés}

11 Si les conflits représentés au fil de la série semblent n'avoir rien de commun avec ceux que nous connaissons, ils établissent pourtant de nombreuses analogies avec des guerres 
contemporaines du XXe siècle, et possèdent un sous-texte qui résonne avec l'Histoire américaine, tout en affichant systématiquement des prétentions universalistes.

\section{La guerre en trame de fond}

12 Le premier conflit mentionné par Babylon 5 est toutefois celui qui rend le moins d'écho, et se trouve en dehors du récit premier : terminé depuis déjà dix ans lorsque commence la série, la courte guerre Terre/Minbar continue d'alimenter les tensions entre les deux peuples, et notamment un climat xénophobe qui sera le terreau des conflits à venir. Toutefois, il s'agit aussi de montrer comment deux peuples peuvent surmonter l'incompréhension, le fossé qui les sépare. La série ne cesse de revenir sur ce conflit via des flashbacks - souvent liés à la mémoire traumatique du commandant Sinclair -, et plus directement via un téléfilm spécial (In the Beginning, Michael Vejar, 1998). Il part d'un incident stupide : lors du premier contact entre les Terriens et les Minbaris, ces derniers déploient les canons de leurs vaisseaux en signe de respect, un acte que les Terriens prennent pour une agression, avant de faire feu.

La série va, durant ses deux premières saisons, construire une ironie dramatique : si les Minbaris, après avoir dévasté les colonies terriennes, se sont arrêté aux portes de la Terre et ont cessé le feu, c'est parce qu'ils ont compris que leurs âmes, dont ils craignaient la " disparition ", se réincarnent aussi chez les Terriens. C'est le secret que le commandant Sinclair va lentement dévoiler, en cherchant à éclairer le mystère de son enlèvement par les Minbaris juste avant la fin du conflit. Les deux peuples qui s'entredéchiraient ne font en réalité qu'un, un tout qui ne peut être que diminué par l'horreur de la guerre, ainsi que l'explique le minbari Lennier dans Points of Departure (2.1):

LENNIER. Remove those souls, and the whole suffers. We are diminished.

Le remplacement de Sinclair par Sheridan au début de la saison 2 va réalimenter les tensions Terre/Minbar, le capitaine étant un héros de guerre parvenu à détruire un croiseur minbari. Mais cette fois-ci, c'est plus directement à l'échelle biographique que les deux peuples surmontent leurs différences, lorsque Sheridan et l'ambassadrice minbarie, Delenn, non seulement entretiennent une relation romantique mais deviennent les deux leaders de "l'Armée de la Lumière » durant le conflit contre les Ombres. Leur alliance et leur relation, symboles d'ouverture et de progrès, se posent en contrepoint du climat xénophobe qui mine peu à peu la Terre. Le fait que Delenn et Sheridan soient deux des personnages majeurs de la série lui permet de porter haut des valeurs d'ouverture, d'acceptation, de communalité, valeurs qui sont toutefois sournoisement minées par l'emphase sur le capitalisme comme modèle économique hégémonique ${ }^{14}$, et par la mise en scène de la démocratie libérale comme objectif idéal, comme « fin de l'Histoire » au sens de Fukuyama ${ }^{15}$.

\section{Les échos de la guerre du Vietnam}

15 Le premier conflit qui éclate dans le présent de la narration est la seconde invasion de la planète Narn par les Centauris, qui conclue la saison 2 après une lente escalade belliqueuse entre les deux peuples. Dans The Long, Twilight Struggle (2.20), les Centauris mettent un point final à leur plan d'invasion en bombardant la planète des Narns avec des mass drivers, des catapultes électromagnétiques dont l'usage est pourtant interdit par les lois galactiques. On peut y lire une critique de l'utilisation de l'arme nucléaire comme des 
bombardements du Japon et du Nord Vietnam par les États-Unis ; le décor aride et chaud de la planète des Narns fait aussi écho à d'autres bombardements : ceux, très médiatisés, qui ont ouvert l'opération Tempête du Désert, en 1991 durant la guerre du Koweït... deux ans avant la diffusion du téléfilm pilote The Gathering (Richard Compton, 1993) ${ }^{16}$. Préfigurant de manière proleptique l'échec de cette invasion centaurie (suite à une guérilla menée par les Narns durant la saison 3), la chaîne d'actualités interstellaire ISN rapporte dans le 2.20 les propos du sénateur Quantrell, qui explique que les Narns ont été "bombed back to the Stone Age ", ramenés à l'âge de pierre par le bombardement centauri.

L'allusion renvoie ici à la guerre du Vietnam, plus précisément à la biographie du général Curtis LeMay publiée en $1965^{17}$, lorsqu'il évoquait par ces mêmes mots la possibilité de paralyser le Vietnam à l'aide de la force aérienne et navale américaine... force qui dut finalement plier bagages en 1975, de même que les Centauris, si certains de leur domination technologique et martiale sur les Narns, en oublient la résilience de ces derniers. Quand bien même la série touche ainsi à l'Histoire américaine de la seconde moitié du XXe siècle, elle sait toutefois conserver un message universel, souvent explicité par les discours des personnages : la plupart occupant de hautes responsabilités, ces longs monologues empreints de gravité apparaissent justifiés. Lorsque, dans The Long, Twilight Struggle (2.20), l'ambassadeur Londo Mollari exige que l'ambassadeur G'Kar soit destitué et fait prisonnier, le Narn se lève en silence et, alors que son peuple meurt sous les bombes, prononce un vœu de liberté devant le Conseil des peuples de la galaxie :

G'KAR. No dictator, no invader can hold an imprisoned population by force of arms forever. There is no greater power in the universe than the need for freedom. Against that power, governments and tyrants and armies cannot stand. The Centauri learned this lesson once. We will teach it to them again. Though it take a thousand years we will be free.

17 Cette scène capitale, soutenue par la bande sonore musicale douce-amère de Christopher Franke, est aussi celle où tombent les masques, et où le plus belliqueux n'est pas l'autre (les Narns reptiliens) mais celui qui nous ressemble (l'ambassadeur Mollari, qui hormis sa coupe de cheveux extravagante, aurait sa place dans l'aristocratie terrienne). Babylon 5 permet par la suite au personnage de G'Kar de comprendre qu'il doit briser le cycle de la violence qui unit les deux peuples, et jouer sur le terrain des Centauris - celui des apparences - pour gagner la liberté de son peuple. S'ensuit une relation complexe avec Mollari qui, reconnaissant ses erreurs, aidera les Narns à retrouver leur liberté.

\section{Les heures sombres de l'histoire américaine récente réinvesties}

De tous les conflits qui agitent le monde fictionnel de la série, les deux plus importants fonctionnent en tandem et structurent le déploiement du récit durant les quatre premières saisons : la Guerre des Ombres, et son pendant plus localisé, la Guerre Civile Terrienne, résultat de la montée d'un gouvernement fasciste au service des Ombres. C'est via ces deux conflits que la série articule l'essentiel de son discours thématique ; le climat terrien est toutefois le plus propice à des allusions constantes à l'Histoire du monde réel.

19 Au fil de la saison 1 sont disséminés des indices récurrents pointant vers le climat xénophobe et isolationniste qui « infecte » peu à peu la Terre : ce n'est pas un hasard si la première mention (par le docteur Franklin) des groupes terriens anti-aliens a lieu dans Infection (1.4), un épisode dont l'intrigue concerne une arme destinée à éradiquer tout organisme considéré comme « impur ». Le groupe Homeguard mène deux attaques contre 
les représentant(e)s aliens $(1.7,1.11)$, et l'armée terrienne s'allie au Psi Corps, le corps des télépathes, pour mener des interrogatoires à la frontière de la légalité dans Eyes (1.16). Cette menace latente est mise au jour à la fin de la saison 1 : Chrysalis (1.22) montre l'assassinat du président Santiago, puis la prise de pouvoir de son vice-président, en usant d'une mise en scène calquée sur les photos d'archives de Lyndon B. Jonson prêtant serment à bord de Air Force One, le 22 novembre 1963, après l'assassinat du président Kennedy. C'est donc un traumatisme médiatisé, une image marquante de l'Histoire américaine, que Babylon 5 réemploie pour assurer la portée de son discours : elle s'adresse d'abord au public américain, et l'accession au pouvoir du vice-président Clark à bord du vaisseau spatial Earthforce Two, médiatisée par un écran, place les personnages dans une posture réflexive de spectateur/ice. Les protagonistes regardent autant un moment d'Histoire que nous regardons une portion de leur histoire... et inversement.

Durant la saison 2, le gouvernement Clark met en place un Ministère de la Paix aux accents orwelliens; ses représentant(e)s n'hésitent pas à employer l'expression « peace in our time ", qui au-delà d'une prière anglicane, évoque le discours du Premier Ministre britannique Neville Chamberlain au retour de la signature des accords de Munich en 1938. Ce pacte de non-agression, ce n'est pas avec l'Allemagne nazie que le Ministère de la Paix le signe, mais c'est tout comme, puisqu'il s'agit des Centauris qui, aidés secrètement par les Ombres, se lancent dans l'invasion et la destruction des avant-postes des Narns. Quand Chamberlain abandonnait la Tchécoslovaquie, la Terre condamne les Narns.

21 Dans un mouvement qui évoque les heures sombres du maccarthysme, et plus largement la montée des fascismes, le Ministère de la Paix est à l'origine de la création d'une milice, Night Watch, dont les membres arborent des brassards noirs qui ne passent pas inaperçus, y compris dans les épisodes (de plus en plus feuilletonnants dès la saison 2) qui ne concernent pas directement les dérives du gouvernement Clark: non content de gagner en continuité et cohérence, le monde fictionnel ploie sous les signes discrets d'une surveillance angoissante à laquelle les personnages principaux, engagés pour la liberté, cherchent à échapper. Science-fiction oblige, la menace monte d'un cran lorsque le Psi Corps, qui rassemble les télépathes possédant une assermentation, se met au service du gouvernement Clark, obligeant les personnages humains à se protéger en s'entourant de télépathes... minbaris (Dust to Dust, 3.6).

Conflit majeur de l'intrigue macroscopique de la série, la Guerre Civile Terrienne qui agite la fin de saison 4 est précédée d'une courte mais sanglante sécession au milieu de la saison 3, lors du triptyque 3.8-3.10. La colonie martienne, dont les troubles constituent depuis le début de la série un sujet d'actualité récurrent, est l'étincelle qui précipite la première étape $\mathrm{du}$ conflit lorsque la planète rouge se rebelle contre l'oppression $\mathrm{du}$ gouvernement Clark et demande son indépendance. Le capitaine Sheridan n'a d'autre choix que de déclarer à son tour l'indépendance de Babylon 5 à la suite de batailles sanglantes contre les forces de Clark. La particularité de ces combats est bien sûr soulignée par le général Hague, allié de Sheridan, qui rappelle que «cette fois-ci, ils connaissent ceux sur qui ils tirent ».

\section{Une dénonciation de la guerre}

23 Après une période consacrée à la Guerre des Ombres, la Guerre Civile Terrienne reprend de plus belle des épisodes 4.15 à 4.21 . Sheridan refuse que sa flotte spatiale ouvre le feu la première lors des combats. Les protagonistes sont bien sûr " du bon côté ", et à mesure 
que le gouvernement Clark commet les pires crimes de guerre, torture Sheridan le temps d'un long épisode se déroulant dans un huis-clos étouffant (Intersections in Real Time, 4.18), et agite une propagande outrancière, la série ne laisse que peu d'espace pour questionner les actions des personnages principaux. C'est à peine si, une fois les forces de Clark vaincues, les actions de Sheridan sont questionnées par la nouvelle présidente, Susanna Luchenko (Beata Pozniak Daniels) : ayant participé au renversement du pouvoir exécutif en place, Sheridan quitte l'armée pour éviter de donner au gouvernement Luchenko les allures d'une dictature militaire. Mais par un habile calcul, il est nommé Président de l'Alliance Interstellaire tout juste formée (un équivalent fictionnel des Nations Unies), dont les vaisseaux survolent Genève dans une démonstration pacifique qui a pourtant tout de l'avertissement martial. La saison 5 se construit ensuite sur un équivalent réducteur de la Reconstruction américaine post-guerre civile, uniquement focalisé sur la recherche d'une unité terrienne brisée par la guerre civile : elle s'incarne, sur Babylon 5, sous les traits de la colonelle Elizabeth Lochley, nommée à la tête de la station, et dont la position durant la guerre civile reste longtemps un mystère, avant qu'elle n'avoue être restée neutre dans Strange Relations (5.6). Avec elle s'éteint petit à petit la problématique de la réconciliation terrienne, sans que sa complexité et l'analogie avec la Reconstruction ne soient jamais vraiment explorées.

24 À l'échelle de la galaxie, c'est une guerre froide qui oppose les deux puissances en présence : les mystérieux Vorlons, paternalistes et faussement bienveillants ; les Ombres, qui procèdent à une sélection tout sauf naturelle des jeunes civilisations, en les poussant à s'entredéchirer. Si les Ombres restent des antagonistes, les Vorlons passent de protagonistes à antagonistes durant la saison 4 : ils ont manipulé l'évolution des jeunes civilisations, et n'hésitent pas à les rayer de la carte s'ils les considèrent " corrompues » par les Ombres. D'une guerre froide au cours de laquelle les deux superpuissances tiraient les ficelles en secret, poussant les jeunes civilisations à se battre au nom de leurs idéologies respectives, on passe en début de saison 4 à un affrontement direct et absurde, une politique de la terre brûlée contre laquelle «l'Armée de la Lumière » va se rebeller. Vorlons et Ombres ne pouvant être détruits par les armes conventionnelles de la future Alliance Interstellaire, ils sont finalement "sermonnés " par le capitaine Sheridan et l'ambassadrice Delenn dans Into the Fire (4.6) : au plus fort de la guerre, alors qu'il ne reste plus aucun espoir, le duo parvient à contacter les deux camps et les place devant leurs contradictions.

SHERIDAN. The Vorlons ask only one question over and over: "who are you?" You [il montre l'Ombre], for you the question is, "what do you want?" I have never heard you answer that question. Who are you? What do you want?

DELENN. You don't know, do you? You've been fighting each other so long, you've forgotten. You've lost your way. So how can you guide us? How can we learn who we are and what we want if you don't even know anymore? [...]

SHERIDAN. You're as trapped in this cycle as much as we are. But we can't afford it anymore. We don't need it. We don't need you. We've learned how to stand on our own. We'll make mistakes, but they'll be our mistakes, not yours.

25 La série cherche là encore à diffuser un message qui se veut universel : elle dénonce les idéologies binaires. Kosh, l'ambassadeur des Vorlons, le dit lui-même:

коSH. Understanding is a three-edged sword: your side, their side, and the truth.

Là où la Guerre Civile Terrienne se résout par les armes, la Guerre des Ombres se termine sur une révélation, une négociation, la naissance d'un Âge nouveau pour les jeunes civilisations de la galaxie. Cette résolution hâtive des deux conflits majeurs de l'intrigue 
macroscopique a ses racines dans le contexte de production de la série : le diffuseur de Babylon 5, PTEN, est voué à disparaitre en 1997, et le plan narratif quinquennal de J. Michael Straczynski est menacé. La saison 4 est alors l'objet d'une lourde reconfiguration pour parer à une annulation. La série est finalement reprise par le network TNT, et se voit accorder la dernière de ses cinq saisons. Celle-ci est alors consacrée aux arcs narratifs laissés en suspens par la reconfiguration, et peut s'étendre, non pas sur la guerre, mais sur le difficile maintien de la paix.

L'Alliance Interstellaire est un projet ambitieux et complexe que peinent à mener Sheridan et Delenn ; une guerre civile éclate sur la planète Minbar, un conflit entre les castes dont Delenn est indirectement responsable, et dont elle doit assumer les conséquences. Enfin, les Centauris, qui s'étaient alliés aux Ombres, se retrouvent manipulés par les alliés revanchards de l'ennemi millénaire. Les Drakhs trouvent en effet refuge sur la planète Centauri Prime et manipulent son gouvernement pour mieux provoquer une guerre interstellaire qui plongera la planète dans une politique isolationniste à même de cacher leurs noirs desseins. Cette guerre, le président Sheridan s'y refuse longtemps, et c'est avec lassitude et dégoût qu'il y engage l'Alliance Interstellaire, sous pression des différents peuples de la galaxie. Dans All My Dreams, Torn Asunder (5.16), il se rend à l'inévitable conclusion devant les représentant(e)s de l'Alliance, non sans expliciter son amertume à en arriver si vite à un tel extrême.

SHERIDAN. Now, we gave you a promise and we are bound by that promise, and damn you for asking for it! And damn me for agreeing to it. And damn all of us to hell, because that is exactly where we're going! We talked about peace. You didn't want peace! We talked about cooperation. You didn't want cooperation! You want war. Is that it? You want a war? Well, you've got a war!

C'est au fil de cette dernière saison, comme lors des bonds dans le futur opérés par War Without End, Part 2 (3.17) et The Deconstruction of Falling Stars (4.22) que la série articule un discours somme toute assez pessimiste: les conflits qui forment la colonne vertébrale de l'intrigue macroscopique de Babylon 5, et dont le dénouement offre une clôture narrative, ne sont que des itérations parmi d'autres, au sein d'un vaste monde fictionnel, pétri de siècles, de millénaires de guerres passées, et de siècles, de millénaires de guerres futures, desquelles le récit donne un aperçu glaçant. Si la série cherche à offrir un message d'espoir, elle se construit sur une vision cyclique du temps et de l'Histoire qui constitue le creuset de son discours thématique.

\section{La mémoire et la place de l'individu dans l'Histoire}

Comme l'explique Mehdi Achouche, la série use d'un «effet-réalité » qui doit à la fois divertir le téléspectateur en l'emportant dans une réalité imaginaire totalisante dont on nous décrit et explicite les aspects aussi bien politiques, historiques, religieux, économiques ou sociaux ; mais aussi très souvent le ramener à la réalité initiale, notre réalité (ou la réalité spécifique de chaque téléspectateur), l'aperçu globalisant offert au téléspectateur omniscient lui permettant de donner du sens à une réalité et un présent souvent perçus de manière fragmentée et partielle ${ }^{18}$. 


\section{Le rôle des médias}

30 Je rejoins aussi Kurt Lancaster lorsqu'il explique que Babylon 5, malgré sa structure aristotélicienne marquée par un début, un milieu et une fin, mobilise plutôt l'épique brechtien. La série cherche en effet à maintenir spectateurs et spectatrices dans une position liminale, entre l'immersion dans la fiction et la prise de recul par rapport aux événements représentés, aux thèmes abordés, et invite à réfléchir non seulement sur les liens entre les conflits fictionnels et leurs équivalents réels, mais plus largement à questionner la construction même de l'Histoire ${ }^{19}$.

31 Babylon $5 \mathrm{a}$, de manière flagrante, pris note de la surmédiatisation des conflits contemporains tels que la Guerre du Golfe. Cette surmédiatisation est répercutée dans le monde fictionnel. Comme l'explique Kurt Lancaster,

The series' characters inhabit a world profoundly shaped by the flow of news and information across various channels of communication. They read about events that affect them in the newspaper or watch them unfold on television. They give interviews to reporters, and we watch as what they say is distorted to serve various agendas ${ }^{20}$.

La série est la première à utiliser, à la télévision, des images de synthèse plutôt que des maquettes pour représenter les vaisseaux et corps célestes. Les scènes de combats spatiaux sont donc très dynamiques, et une importance cruciale est accordée à l'élaboration de leur stratégie par les personnages: chaque escarmouche ne se résume pas à un échange de tirs mais exploite l'espace dans toutes ses dimensions. Si cette technique permet de réduire le coût d'un épisode ${ }^{21}$, la narration ne peut pas toujours justifier d'incessants allers-retours entre la station Babylon 5, cœur de l'action, et le reste de la galaxie où sont livrées les batailles, puisque les protagonistes sont censés rester à leur poste.

32 Ainsi la série va-t-elle très fréquemment utiliser les reportages de la chaîne d'actualités fictive ISN pour informer personnages et spectateur/ices de ce qui se passe ailleurs dans le monde fictionnel, tout en imposant la station diplomatique comme le canal où transitent les informations autant que les populations. Dans un tournant réflexif, on voit donc souvent les protagonistes regarder la télévision pour assister à des reportages et flashs d'informations. Ils n'ont rien d'un fond sonore, puisqu'ils ouvrent ou concluent souvent une scène ou l'acte d'un épisode, en dévoilant de façon partielle comment une situation qui a touché la station a été perçue ou s'est conclue ailleurs dans la galaxie; ou comment une situation initiée ailleurs dans la galaxie va avoir des répercussions sur la station.

C'est notamment la chaîne ISN qui devient bientôt le témoin privilégié de la prise de pouvoir du président Clark, et si elle est muselée durant la saison 2, elle est au cœur des événements précédant la sécession en saison 3. Une scène capitale de Severed Dreams (3.10), qui conclue le triptyque de la sécession, se déroule sur les plateaux d'ISN bombardés par les milices de Clark. La série se permet alors de traverser la frontière de l'écran pour transporter le récit sur Terre, au moment où une journaliste est interrompue par un collègue avouant aux téléspectateur/ices que diverses colonies ont déclaré leur sécession de l'Alliance Terrienne, en réponse à la promulgation de la loi martiale sur Mars. Via un travelling arrière circulaire, la caméra incorpore les coulisses dans le cadre, et l'on peut alors voir l'agitation dans les studios, avant que le son d'une fusillade ne se 
fasse entendre, et que l'image ne soit coupée. Une fois ISN tombée aux mains du pouvoir, diffusant des messages propagandistes et ne disant que ce que le gouvernement Clark veut entendre, les personnages ne s'en désintéresseront pas pour autant, réagissant furieusement aux mensonges proférés par les journalistes, de la "Menace Rouge» représentée par la colonie martienne aux contre-vérités sur le déroulement de la Guerre des Ombres.

À deux reprises, Babylon 5 accueille une équipe de journaliste : avant la sécession (2.15), où déjà le montage final modifie les propos donnés en interview pour faire passer Sheridan et les ambassadeurs pour de dangereux lunatiques; puis après la sécession, en saison 4 (4.8). Les protagonistes ne semblent pas avoir appris de leur erreur : cherchant à utiliser les médias pour prouver qu'ils ne sont pas les ennemis de la Terre, ils seront au contraire montrés sous leur plus mauvais profil, les journalistes à la solde du pouvoir déformant la réalité pour motiver un assaut final contre Babylon 5 dans l'opinion publique.

Toutefois, ainsi que le relève Sherryl Vint, si la série montre à de nombreuses reprises comment l'information peut être manipulée,

Babylon 5 avoids pursuing the full implications of its critique - that any story, including its own narrative, is ideology from an interested position preferring instead to assert a firm binary relationship between truth and propaganda ${ }^{22}$.

Ainsi les journalistes qui montent à bord de la station sont-ils présentés sans ambiguïté comme les instruments du pouvoir fasciste de Clark, et les présentatrices des actualités d'ISN clairement catégorisées : respectant la continuité, la série emploie principalement deux actrices, l'une avant la sécession et après la libération (Maggie Egan), l'autre pendant les débordements du gouvernement Clark (Diana Morgan), les associant dans la mémoire du public l'une à la vérité, l'autre à la propagande.

\section{Le témoin et l'oubli}

Face à des médias omniprésents qui peinent à véhiculer la vérité, Sheridan commence à comprendre - comme G'Kar l'avait compris dans le cadre du conflit Narns-Centauris - que la guerre se joue aussi en termes d'images. Il met en place une émission diffusée dans toute la galaxie, intitulée Voice of the Resistance, afin de diffuser les informations sur le conflit contre les Ombres, puis coordonner les informations de la flotte sécessionniste. Ironie du sort, dans le bien-nommé Rumors, Bargains and Lies (4.13), il demande à sa commandante en second Ivanova de mentir en précisant dans une occurrence de Voice of the Resistance que « rien ne s'est passé aujourd'hui» dans un secteur donné de la galaxie, afin de provoquer une réaction de la part des Mondes Non-Alignés qui refusent encore d'assister les sécessionnistes dans le conflit à venir contre la Terre. Le public peut alors partager l'incompréhension d'Ivanova lorsque Sheridan lui donne cet ordre juste avant la diffusion de l'émission :

SHERIDAN. I just want you to mention that nothing happened today in Sector 83 by 9

by 12 .

IVANOVA. 8-3-9-1-2?

SHERIDAN. That's the one.

IVANOVA. But, sir, I just got a status report from C \& $C$ a little while ago and that

sector's been absolutely quiet. 
SHERIDAN. Exactly. So just relax, because what you're saying is absolutely true.

IVANOVA. That nothing happened there today?

SHERIDAN. Yes.

IVANOVA. Just casually mention that nothing's going on.

SHERIDAN. That's right. Make a big deal out of it as much as you can, given nothing

happened.

IVANOVA. Sir, I-

SHERIDAN. 10 seconds. I better get out of the way. You're about to go on.

Au-delà d'une référence à la phrase (supposée) que le roi George III aurait consignée dans son journal le 4 juillet $1776^{23}$, jour de l'Indépendance des États-Unis, la série use ici des faiblesses de ses personnages, qui ne sont jamais blancs comme neige, ainsi que le relève Mehdi Achouche $^{24}$. Les protagonistes doivent parfois mentir, voire sacrifier leurs subordonnés (notamment le ranger Ericsson, dont la mission-suicide est vitale pour la victoire finale sur les Ombres dans The Long Night, 4. 5) ; les conflits moraux ont autant d'importance que les conflits physiques, notamment lorsqu'ils portent sur la rétention d'informations, les énigmes et les secrets qui alimentent les arcs narratifs de la série. Sheridan cite par exemple le bombardement de Coventry durant la Seconde Guerre Mondiale, et le mythe qui l'entoure ${ }^{25}$, pour se convaincre lui-même qu'il doit garder le secret quant à l'existence des Ombres (In the Shadow of Z'ha'dum, 2.16).

Mais il y a pire crime dans le monde fictionnel que de tordre ou cacher les faits : c'est de les oublier. Au-delà de la nécessité de véhiculer une information la plus objective possible, la série insiste, tout au long de ses cinq saisons, sur le devoir de mémoire et sur le caractère néfaste de l'oubli. D'autres médias plus anciens sont la clé des victoires présentes, notamment le Livre de G'Quan, un ouvrage narn que l'ambassadeur G'Kar confie à Garibaldi : en le déchiffrant, le chef de la sécurité découvre des informations capitales sur les Ombres, récoltées lors d'une précédente guerre, 1000 ans plus tôt. Dans cette série de science-fiction mâtinée de fantasy, l'ancien livre saint détonne à peine dans le centre de commande futuriste, et les légendes se confondent avec les faits historiques. Les guerres qui parsèment l'Histoire du monde fictionnel sont toujours des repères fondamentaux, et, comme nous le verrons bientôt, même les événements de la série deviendront, dans le futur du monde fictionnel, des faits historiques parfois déformés, et même des légendes.

Au-delà des conflits majeurs, la série ne cesse de jouer sur le thème de la mémoire. Les Drazis, qui forment un peuple mineur mais toujours présent en arrière-plan, mènent de façon cyclique un combat à mort entre deux factions désignées de façon arbitraire pour élire un nouveau leader (The Geometry of Shadows, 2.3); l'origine de cette coutume est perdue dans les siècles passés. Les Hyachs, dans Epiphanies (4.7), se sont forcés à oublier le génocide qu'ils ont commis sur une espèce cousine de la leur, alors même que ce génocide a condamné leur propre espèce, empêchant tout brassage génétique et les tuant à petit feu. Devant ces oublis impardonnables, certaines civilisations se sont fait un devoir de conserver les savoirs et l'Histoire de la galaxie, et elles fuient les Ombres pour préserver leur savoir: les technomages (2.3), les Vindrizis (Exogenesis, 3.7), ou encore les Soul Hunters qui emprisonnent les âmes des personnalités majeures de diverses civilisation, pour conserver intacts leur souvenir (Soul Hunter, 1.2).

Les témoignages d'ordre plus individuels peuvent aussi avoir une influence cruciale sur le déroulement de l'Histoire: ainsi l'arrivée de l'archéologue venue révéler aux protagonistes la découverte d'un vaisseau des Ombres sur Mars est-elle filmée avec emphase, comme s'il s'agissait d'une personnalité publique escortée par des agents de 
sécurité, alors que les spectateur.ice.s n'ont jamais vu ce personnage auparavant ( Messages from Earth, 3.8). Le souvenir perdu de Sinclair, premier commandant de Babylon 5 , qui a oublié sa rencontre avec les Minbaris lors de la dernière Bataille dans la guerre Terre/Minbar, est également un enjeu narratif majeur de la première saison. microscopique des épisodes, les problématiques déployées par la matrice de la série. Ainsi il apparait que l'un des ingrédients principaux de la formule de la série - un de ses points nodaux ${ }^{26}$ répétés de façon systématique dans la majorité des épisodes - consiste en la confrontation d'un personnage - un protagoniste ou un personnage secondaire - à un élément de son passé. Dans l'épisode Passing Through Gethsemane (3.4), un tueur en série, condamné autrefois à avoir la mémoire effacée et à être reprogrammé - il est devenu moine - commence à se souvenir, et s'interroge : comment peut-il demander pardon pour ses crimes s'il les a oubliés? Dans A Late Delivery from Avalon (3.13), le soldat qui, le premier, a tiré sur les Minbaris par erreur, débutant la guerre Terre/Minbar, est atteint d'un syndrome de stress post-traumatique et se prend pour le roi Arthur, incapable de faire face à son acte aux conséquences dévastatrices. Tout au long de la série, les personnages principaux font systématiquement face à des connaissances de leur passé, leur rappelant leurs deuils, leurs erreurs, et les obligeant à faire face à leur conscience et leurs dilemmes moraux ${ }^{27}$.

\section{La construction du récit historique et la figure du héros}

Babylon 5 aborde plus directement la construction de la mémoire historique. Les personnages ne cessent ainsi de s'interroger sur leur place dans l'Histoire, à mesure que leurs actions influent sur de plus en plus de vies à travers la galaxie. Sheridan cite Abraham Lincoln dans son discours de Points of Departure (2.1) (" We cannot escape history. We will be remembered in spite of ourselves. »); l'équipe craint de modifier l'Histoire en remontant dans le temps dans le diptyque War Without End $(3.16,3.17)^{28}$; le terrifiant Morden justifie l'idéologie des Ombres en mentionnant les guerres qui parsèment les 6000 ans d'Histoire écrite de l'humanité ( $\left.Z^{\prime} h a^{\prime} d u m, 3.22\right)$.

Mais c'est surtout l'épisode The Deconstruction of Falling Stars (4.22) qui cristallise le propos de la série. Écrit en catastrophe pour remplacer un épisode conclusif ${ }^{29}$, lorsque la série est finalement renouvelée pour une cinquième saison, The Deconstruction... est un épisode indépendant qui met en scène un être du futur consultant des archives vidéo, disséminées à quatre époques différentes : 2262, un talk-show diffusé juste après la victoire des forces de Sheridan contre le gouvernement Clark ; 2362, l'équivalent d'un MOOC, un cours en ligne où des universitaires dissertent sur l'héritage laissé par Sheridan, Delenn et l'Alliance Interstellaire ; 2762, année durant laquelle la Terre est de nouveau soumise à une dévastatrice guerre civile, et l'un des deux camps manipule des hologrammes représentant les protagonistes de la série afin de les faire passer pour des monstres sanguinaires; enfin, 3262, des vidéos de surveillance dissimulées chez des moines œuvrant pour la reconstruction de la Terre, revenue à un état technologique préindustriel 500 ans après la guerre dévastatrice de 2762.

Ainsi que le souligne James Brown, The Deconstruction of Falling Stars articule un thème qui domine toute la série: la capacité d'un individu à marquer l'Histoire ${ }^{30}$. Le premier segment est le plus à même de relativiser les intentions de Sheridan au sortir de la Guerre Civile, et de questionner le portrait du héros de guerre dressé par les colonies libérées. 
Toujours selon Brown, le segment du MOOC du futur est peut-être le "moins ambigu » puisqu'il « oriente la sympathie du public ${ }^{31}$ » vers Sheridan - décédé depuis longtemps et Delenn, âgée mais toujours vive, qui intervient en sa faveur: les universitaires se montrent en effet très critiques envers cette conception de l'Histoire qui valorise l'influence de l'individu. Lorsqu'on écoute le commentaire audio de l'épisode disponible sur l'édition DVD de la série, il est d'autant plus évident que J. Michael Straczynski défend une vision individualiste, et la figure du héros, et ce au moment même où, comme le relèvent Edward James et Farah Mendlesohn, la « narrative history » fait son retour dans la sphère académique américaine des années 1990, en opposition à une conception marxiste de l'Histoire (défendue par les universitaires de l'épisode) selon laquelle Sheridan et Delenn ont pris le train de l'Histoire en route, et leurs rôles auraient pu être tenus par d'autres ${ }^{32}$.

Il faut toutefois noter que le dernier segment de l'épisode relativise cette figure du héros : les protagonistes sont devenus des icônes divines au milieu des enluminures des vieux ouvrages consultés par les deux moines. Leur biographie et leurs actes y sont autrement plus manichéens et simplistes que dans le reste de la série : rien n'est dit, par exemple, de la trajectoire problématique de Delenn - les accents de fanatisme religieux qui la mèneront à briser le système des castes minbaries et provoquer une guerre civile sur sa planète; ou des actions discutables de Sheridan envers les télépathes durant la saison 5 , actions qui amorceront une guerre future que la série ne fait que mentionner sans jamais l'explorer. James et Mendlesohn notent aussi que la dimension épique du récit remet en question le libre-arbitre de ces figures héroïques face aux entités « divines » que sont les Vorlons et les $\mathrm{Ombres}^{33}$. Au centre des multiples conflits de la série, il faut souligner à la suite de Sherryl Vint que

Babylon 5 struggles with it simultaneous desires to be a novel for television including characteristics of the novel such as complex and flawed characters - and its epic narrative arc, which conventionally requires glorification of the hero and a clear victory for the right side ${ }^{34}$.

\section{Conclusion : une leçon d'Histoire américaine}

La série semble ainsi, encore une fois, piégée par ses ambitions : cette glorification du héros, cette vision individualiste de l'Histoire, couplée à son absence de questionnement sur l'hégémonie du capitalisme et de la démocratie libérale au sein de toute la galaxie, et ses références principalement anglo-saxonnes, en font un objet situé dans un contexte de production déterminé. De même qu'au cinéma, autre industrie culturelle hollywoodienne, Babylon 5, série de science-fiction, est "par excellence le lieu de la critique sociale et politique » mais dans le même temps, cette critique n'est « tolérée que pour autant qu'elle entre dans ce que [la] société et ses membres peuvent supporter ${ }^{35}$.» Malgré cela, il faut reconnaître à la suite de Jan Johnson-Smith que

[...] Babylon 5 parle fondamentalement de la connaissance de sa propre Histoire, de la façon dont on doit y rester fidèle, apprendre d'elle, sans quoi l'on est condamné à la répéter - ou à ce qu'elle soit utilisée contre nous. [...] dans Babylon 5, l'Histoire est montrée comme ayant un motif répétitif, et beaucoup de commentaires et éléments périphériques au sein de la série deviennent terriblement évidents lorsque lus rétrospectivement ${ }^{36}$. 
La série ne cherche pas seulement à réitérer cette maxime, mais à questionner ses implications : elle nous fait une leçon d'Histoire, certes américaine et hégémonique bien qu'à vocation universaliste, mais elle s'interroge aussi sur les modalités du devoir de mémoire, et notre rapport au passé, dans une perspective historiographique. Dès Infection (1.4), à propos de l'épuration ethnique perpétrée par les Ikarrans, Ivanova cite George Santayana: «Those who do not remember the past are condemned to repeat it. » Il s'agit là de la question majeure de la série : face à notre monde tristement riche de siècles de guerres, elle anticipe un futur tout aussi sombre, mais dans le même temps, via ses protagonistes engagé(e)s, elle articule un message d'espoir, et nous demande, à nous spectateur/ices, si nous serons un jour capable de briser le cycle éternel des conflits. Ayant poussé la complexité narrative dans ses retranchements, Babylon 5 a eu une influence considérable sur les séries de science-fiction qui ont suivi, notamment au sein du genre space opera. L'ironie du sort veut que son héritière, la "réimagination » de Battlestar Galactica (Syfy, 2003-2009) répète la même question, comme en écho, soulignant un cycle de violence dont on ne peut qu'espérer qu'il ne soit pas infini...

\section{BIBLIOGRAPHIE}

ACHOUCHE Mehdi, « De Babylon 5 à Galactica : la nouvelle science-fiction télévisuelle et l'effet-

réalité », TV/Series, $\mathrm{n}^{\circ}$ 1, juin 2012, p. 311-328

BESSON Anne, D'Asimov à Tolkien: Cycles et séries dans la littérature de genre, Paris, CNRS-Editions, 2004

BROWN James, « Cyborgs and Symbionts: Technology, Politics and Identity », in The Parliament of Dreams: Conferring on Babylon 5, éd. Edward James et Farah Mendlesohn, Reading, Science Fiction Foundation, 1998, p. 110-129

DOLEŽEL Lubomír, Heterocosmica: Fiction and Possible Worlds, Baltimore, Londres, John Hopkins University Press, 1998

DUFOUR Éric, Le Cinéma de science-fiction, Paris, Armand Colin, 2011

ESQUENAZI Jean-Pierre, Les Séries télévisées : L’Avenir du cinéma ?, Paris, Armand Colin, 2010

GENETTE Gérard, Discours du récit, Paris, Points Seuil, 1972, 1983 (édition remaniée 2007)

JAMES Edward, Farah Mendlesohn, « Rewriting the Past in the Future in Straczynski's Babylon 5 », in The Parliament of Dreams: Conferring on Babylon 5, éd. Edward James et Farah Mendlesohn, Reading, Science Fiction Foundation, 1998, p. 1-14

JOHNSON-SMITH Jan, American science fiction TV: Star Trek, Stargate and beyond, Londres, New York, I.B. Tauris, 2004

KUPPERS Petra, «Quality Science Fiction: Babylon 5's Metatextual Universe », in Cult Television, éd. Sarah Gwellian-Jones et Roberta E. Pearson, Londres, Minneapolis, University of Minnesota Press, 2004, p. 45-59 
LANCASTER Kurt, Interacting with Babylon 5: Fan performances in a media universe, Austin, University of Texas Press, 2001

LANE Andy, The Babylon File, Londres, Virgin Publishing, 1997

MCCARRON Kevin, « Religion, Philosophy and the End of History », in The Parliament of Dreams:

Conferring on Babylon 5, éd. Edward James et Farah Mendlesohn, Reading, Science Fiction

Foundation, 1998, p. 131-144

MITTELL Jason, Complex TV: The Poetics of Contemporary Television Storytelling, New York, Londres, New York University Press, 2015

SHU-SHUN CHAN Herbert, « Space and Time Out of Joint: "War Without End" », in The Parliament of Dreams: Conferring on Babylon 5, éd. Edward James et Farah Mendlesohn, Reading, Science Fiction Foundation, 1998, p. 26-37

SOULEz Guillaume, «La Double répétition », Mise au point, n 3, 2011, accessible en ligne à l'adresse http://map.revues.org/979

VINT Sherryl, «Our First, Best Hope for Mature Science Fiction Television », in The Essential Science Fiction Television Reader, éd. J. P. Telotte, Lexington, University Press of Kentucky, 2008, p. 247-265

\section{NOTES}

1. Jan Johnson-Smith, American science fiction TV : Star Trek, Stargate and beyond, Londres, New York, I.B. Tauris, 2004, p. 69.

2. Voir par exemple Jason Mittell, Complex TV : The Poetics of Contemporary Television Storytelling, New York, Londres, New York University Press, 2015, p. 18.

3. L'intention maintes fois affichée du créateur de la série, J. Michael Straczynski, était d'ailleurs de «faire pour la SF télévisée ce que Hill street Blues a fait pour les séries policières » (je traduis ici un de ses très nombreux échanges avec les fans, accessible via les archives du site JMSnews à l'adresse http://www.jmsnews.com/msg.aspx?id=1-21388, consulté le 17 novembre 2015).

4. Straczynski explique que selon ses estimations, cette "histoire racontée en 110 épisodes » occupe «à peu près 5.500 pages [de script] ». Joseph Michael Straczynski, "The Profession of Science Fiction, 48 : Approaching Babylon », Foundation, n64, été 1995, p. 13, reproduit dans Andy Lane, The Babylon File, Londres, Virgin Publishing, 1997, p. 26. Sur le cycle, voir Anne Besson, D'Asimov à Tolkien: Cycles et séries dans la littérature de genre, Paris, CNRS-Editions, 2004, p. 207-208.

5. Sur la constitution de cette figure d'auteur et sa dimension légitimante, on consultera notamment Petra Kuppers, "Quality Science Fiction : Babylon 5's Metatextual Universe », in Cult Television, éd. Sarah Gwellian-Jones et Roberta E. Pearson, Londres, Minneapolis, University of Minnesota Press, 2004, p. 46-48 [p. 45-59].

6. Quand bien même le plan original a été progressivement remanié.

7. Éric Dufour, Le Cinéma de science-fiction, Paris, Armand Colin, 2011, p. 198-199.

8. «[...] le noyau sémantique et symbolique qui commande le récit et son horizon moral ou philosophique » selon la définition de Guillaume Soulez, «La Double répétition », Mise au point, $\mathrm{n}$ • 3, 2011, paragraphe 8, accessible en ligne à l'adresse http://map.revues.org/979.

9. Usant d'une perspective narratologique, je me permettrai d'user du $\mathrm{H}$ majuscule pour distinguer l'Histoire comme discipline, et l'histoire comme « ce qui se déroule dans la diégèse ».

10. Mehdi Achouche, « De Babylon 5 à Galactica : la nouvelle science-fiction télévisuelle et l'effetréalité », TV/Series, $n^{\circ}$ 1, juin 2012, p. 314 [p. 311-328]. 
11. Lubomír Doležel, Heterocosmica: Fiction and Possible Worlds, Baltimore, Londres, John Hopkins University Press, 1998, p. 186 et suivantes.

12. "Any sufficiently advanced technology is indistinguishable from magic. " Première mention dans l'édition révisée de Profiles of the Future, Londres, MacMillan, 1973.

13. Au sens de Gérard Genette, Discours du récit, Paris, Points Seuil, 1972, 1983 (édition remaniée 2007), p. 39. Chaque saison couvre un an d'histoire, ainsi la série se focalise-t-elle sur les années 2258 à 2262, avec des prolepses dont la portée atteint jusqu'à un million d'années dans le futur.

14. Voir notamment la façon dont Kevin McCarron exploite cette idée de démocratie libérale comme «fin de l'évolution idéologique humaine » selon Francis Fukuyama (The End of History and the Last Man, Free Press, 1992), idée qui se retrouve incorporée dans Babylon 5, dont le générique de la saison 4 mentionne d'ailleurs de façon troublante "the end of history". "Religion, Philosophy and the End of History", in The Parliament of Dreams: Conferring on Babylon 5, éd. Edward James et Farah Mendlesohn, Reading, Science Fiction Foundation, 1998, p. 134 et suivantes [p. 131-144].

15. Sherryl Vint insiste notamment sur cette contradiction idéologique de la série. Voir "Our First, Best Hope for Mature Science Fiction Television ", in The Essential Science Fiction Television Reader, éd. J. P. Telotte, Lexington, University Press of Kentucky, 2008, p. 261 [p. 247-265]

16. La série a pour épisode pilote un téléfilm diffusé le 22 février 1993 sur PTEN, et qui raconte la mise en service de la station.

17. Mission With LeMay: My Story, éditions Doubleday.

18. Mehdi Achouche, op. cit., p. 330.

19. Kurt Lancaster, Interacting with Babylon 5: Fan performances in a media universe, Austin, University of Texas Press, 2001, p. 13-15.

20. Ibid., pp. xv-xvi.

21. Jan Johnson-Smith rappelle que la série est deux à trois fois moins chère à produire que ses contemporaines, telles que Space: Above and Beyond (Fox, 1995-1996) ou Star Trek: Voyager (UPN, 1995-2001). Voir op. cit., p. 65.

22. Sherryl Vint, op. cit., p. 261.

23. "Nothing important happened today».

24. Mehdi Achouche, op. cit., 2012, p. 329.

25. La légende (urbaine) suppose que Churchill était au courant du bombardement à venir, mais aurait choisi de ne pas intervenir pour ne pas révéler à l'Allemagne nazie que les Alliés avaient déchiffré le code de la machine Enigma. C'est ainsi qu'elle est rapportée dans l'épisode.

26. Jean-Pierre Esquenazi, Les Séries télévisées: L’Avenir du cinéma?, Paris, Armand Colin, 2010, p. 105.

27. ${ }^{5}$, op. cit., p. 54 .

28. Herbert Shu-Shun Chan note qu'il est évident dès le début du diptyque que les personnages ont littéralement pour mission de protéger l'Histoire, d'autant qu'ils en font alors partie : ils sont l'Histoire, le passé, le présent et le futur du monde fictionnel ainsi que le relève le mystérieux Zathras. Voir "Space and Time Out of Joint: "War Without End" », in The Parliament of Dreams: Conferring on Babylon 5, éd. Edward James et Farah Mendlesohn, Reading, Science Fiction Foundation, 1998, p. 29 [p. 26-37].

29. Qui constitue l'épilogue de la série, et sera finalement diffuse là où il était prévu à l'origine, à la fin de la cinquième saison.

30. James Brown, «Cyborgs and Symbionts : Technology, Politics and Identity », in The Parliament of Dreams: Conferring on Babylon 5, éd. Edward James et Farah Mendlesohn, Reading, Science Fiction Foundation, 1998, p. 121 [p. 110-129].

31. Ibid., p. 122. 
32. Edward James, Farah Mendlesohn, « Rewriting the Past in the Future in Straczynski's Babylon 5 ", in The Parliament of Dreams : Conferring on Babylon 5, éd. Edward James et Farah Mendlesohn, Reading, Science Fiction Foundation, 1998, p. 4 [p. 1-14].

33. Ibid., p. 5-6.

34. Sherryl Vint, op. cit., p. 253.

35. Éric Dufour, op. cit., p. 189.

36. Jan Johnson-Smith, op. cit., p. 206, 209.

\section{RÉSUMÉS}

Cet article explore la représentation des conflits dans la série de science-fiction américaine Babylon 5 (PTEN>TNT, 1993-1998). Dans une perspective narratologique thématique, il s'agit en premier lieu d'analyser le réseau de conflits qui structure le monde fictionnel et l'intrigue de la série au sein d'un récit complexe et non linéaire, ainsi que les analogies tissées de façon systématique avec des conflits réels, dans l'ordre chronologique de la série. Le rapport de la série à l'Histoire, au devoir de mémoire et à l'historiographie est ensuite étudié, au travers de son utilisation des médias évoquant la couverture de la Guerre du Golfe ; de sa dénonciation de la propagande; de l'importance des témoignages historiques pour l'intrigue ; ou encore de la figure du héros brandie par sa forme épique, puis malmenée de façon réflexive. Il est ainsi possible d'explorer les limites de la critique politique et sociale proposée par la série, et de mettre au jour le caractère cyclique de l'Histoire au cœur de son discours.

This paper deals with the representation of war and conflicts in American science fiction tv series Babylon 5 (1993-1998, PTEN>TNT). Following a narratological frame, we first explore the dense net of conflicts structuring the fictional world and the series long term and non-linear plot, along with the systematic comparisons to real world conflicts, in the chronological order of the story. We then study the way the series treats subjects such as History, the need to remember the past, or even historiography, through its use of large-scale medias evoking the live coverage of the Gulf War; its denunciation of propaganda; the importance of historical testimonies for the plot; or the treatment of the hero figure summoned by the epic dimension of the text, and then criticized in a reflexive way. It is then possible to test the limits of the series social and political comment, and to underline the cyclic property of History in the fictional world.

\section{INDEX}

Mots-clés : science-fiction, histoire, cycle, mémoire, narration

Keywords : science fiction, history, cycle, memory, narration

\section{AUTEUR}

\section{FLORENT FAVARD}

Docteur en arts, spécialisé dans les études cinématographiques et audiovisuelles, Florent Favard a soutenu en 2015 à l'université de Bordeaux 3 une thèse intitulée « La Promesse d'un 
dénouement : énigmes, quêtes et voyages dans le temps dans les séries télévisées de sciencefiction contemporaines ». Membre de GUEST-Normandie, il poursuit actuellement ses recherches dans une perspective narratologique contextualiste et transmédiatique focalisée sur les genres de l'imaginaire. Il s'intéresse plus largement aux évolutions du récit et aux problématiques socioculturelles qui y sont liées. 